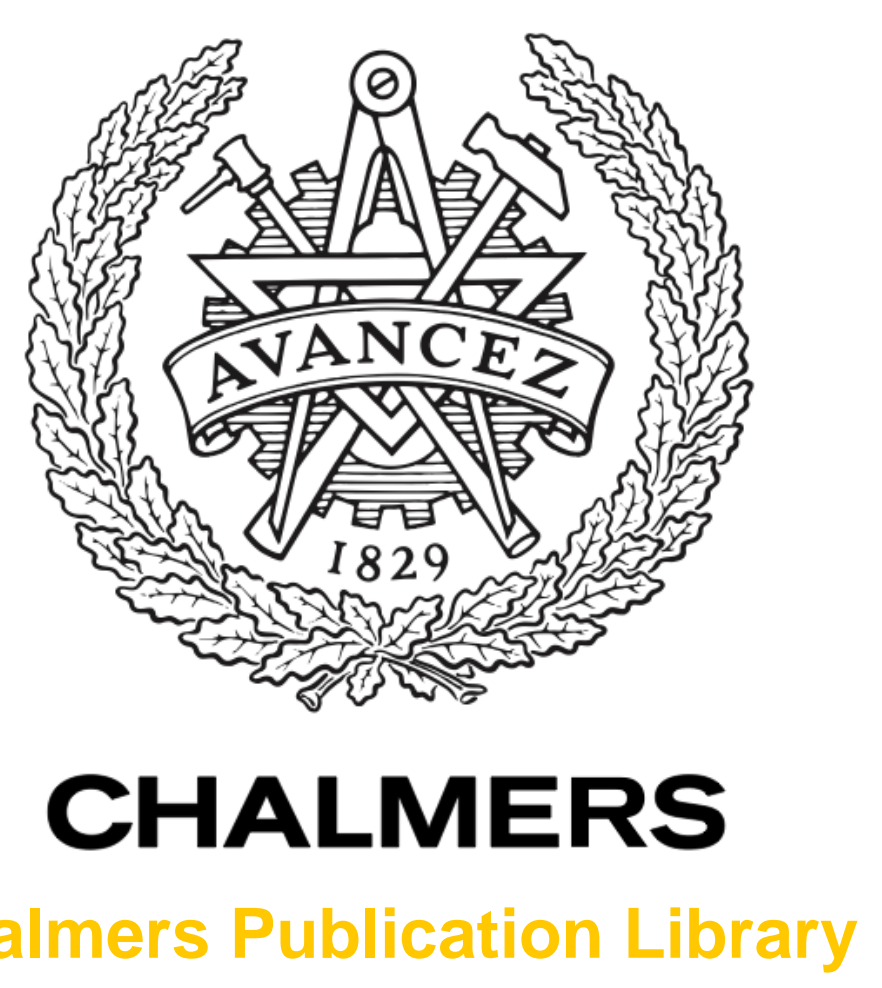

Challmers Publication Library

\title{
Capacity characterization of Eleven antenna in different configurations for MIMO applications using reverberation chamber
}

This document has been downloaded from Chalmers Publication Library (CPL). It is the author's version of a work that was accepted for publication in:

Proceedings of 6th European Conference on Antennas and Propagation, EuCAP 2012. Prague, 26-30 March 2012

Citation for the published paper:

Chen, X. ; Kildal, P. ; Yang, J. (2012) "Capacity characterization of Eleven antenna in different configurations for MIMO applications using reverberation chamber". Proceedings of 6th European Conference on Antennas and Propagation, EuCAP 2012. Prague, 26-30 March 2012 pp. 1571-1575.

http://dx.doi.org/10.1109/EuCAP.2012.6206064

Downloaded from: http://publications.lib.chalmers.se/publication/156663

Notice: Changes introduced as a result of publishing processes such as copy-editing and formatting may not be reflected in this document. For a definitive version of this work, please refer to the published source. Please note that access to the published version might require a subscription. 


\section{Capacity Characterization of Eleven Antenna in Different Configurations for MIMO Applications Using Reverberation Chamber}

\author{
Xiaoming Chen, Per-Simon Kildal, Jian Yang \\ Department of Signals and Systems \\ Chalmers University of Technology \\ Gothenburg, Sweden
}

\author{
Jan Carlsson \\ Electronics department \\ SP Technical Research Institute of Sweden \\ Boras, Sweden
}

\begin{abstract}
In this paper, we characterize the multiple-input multiple-output (MIMO) performance of a multi-port wideband receive antenna with three ideal (i. e. unity embedded radiation efficiency and no correlation) transmit antennas. Different configurations (feeding networks) of the antenna under test (AUT) are examined by measurements in a reverberation chamber. These configurations are studied in terms of embedded radiation efficiencies, correlations, and ergodic MIMO capacities.
\end{abstract}

\section{INTRODUCTION}

Multiple-input multiple-output (MIMO) systems use multiple antennas at both receive and transmit sides to enhance communication performances [1]. A benchmark parameter for the characterization of a MIMO system is the capacity [2], which is also a popular characterization parameter for multi-port antennas from the antenna point-ofview. A lot of studies have been carried out for measuring MIMO capacity in real-life (outdoor and indoor) multipath environments [3]-[7]. Real-life measurements are nonrepeatable, costly and time-consuming. Therefore, as an alternative, reverberation chambers are getting more and more popular in MIMO terminal characterizations, because they provide fast and repeatable measurements for a much lower cost [8]-[15].

A reverberation chamber is basically a metal cavity that is large enough to support many resonant modes. Ideally all of these modes are stirred by mode-stirrers inside the chamber to create many independent samples of linear independent combinations of these modes for good measurement accuracy. The chamber used in this work is the Bluetest reverberation chamber (see Fig. 1), which has been used to characterize MIMO capacities, diversity gains and other performance metrics of different multi-antenna systems, as described in [8][11]. As shown in Fig. 1, the chamber has two plate modestirrers, a turn-table platform, and three antennas mounted on three different walls (referred to as the wall antennas hereafter). The wall antennas are actually wideband half-bowtie (or triangular sheet) antennas [16].

The antenna under test (AUT) used here is the so-called Eleven antenna, which is a dual polarized log-periodic folded dipole array with a frequency band of 2-13 GHz (see Fig. 2) [17]. The Eleven antenna has eight feeding ports, where four ports for one polarization is marked as ports 1-4. The remaining four ports are for the other polarization. In this paper, only ports1-4 are used. Obviously, these ports can be combined in different ways to form different multi-port antennas. Due to the page limitation, only two configurations (specified in Section II) are measured and studied in this paper. The measurement setup is described in Section III-A, measured embedded radiation efficiencies, correlation coefficients, diversity measures, and ergodic capacities of these two Eleven antenna configurations are formulated and examined in Section III-B. Conclusions are given in Section IV.

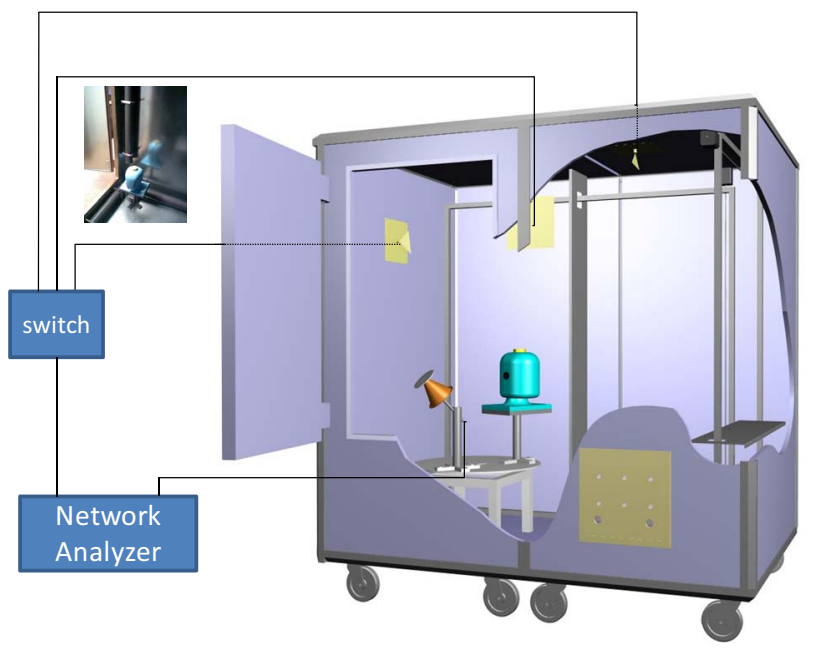

Fig. 1. Drawing of Bluetest RC with two mechanical plate stirrers, one platform, and three wall antennas.

\section{CONFIGURATIONS OF ELEVEN ANTENNA}

The multi-port property of the Eleven antenna enables many possible antenna configurations (depending on the feeding network). Due to the page limitation, only two alternative configurations are investigated in the present paper:

- configuration a (referred to as two-port Eleven antenna): the four ports for one polarization of the eleven antenna (see Fig. 2), are combined to two ports as shown in Fig. 3a by using two wideband $180^{\circ}$ hybrids. 
- configuration $b$ (referred to as three-port Eleven antenna): the four ports for one polarization of the eleven antenna (see Fig. 2), are combined to three ports as shown in Fig. $3 \mathrm{~b}$ by using one wideband $180^{\circ}$ hybrid.

The two-port Eleven antenna has been used in [10] for determining capacity from measurements over large bandwidth in both reverberation and anechoic chambers. In the present paper, we include it as a reference and compare its MIMO performance with that of the three-port Eleven antenna. In addition, their embedded radiation efficiencies and correlations are also compared.

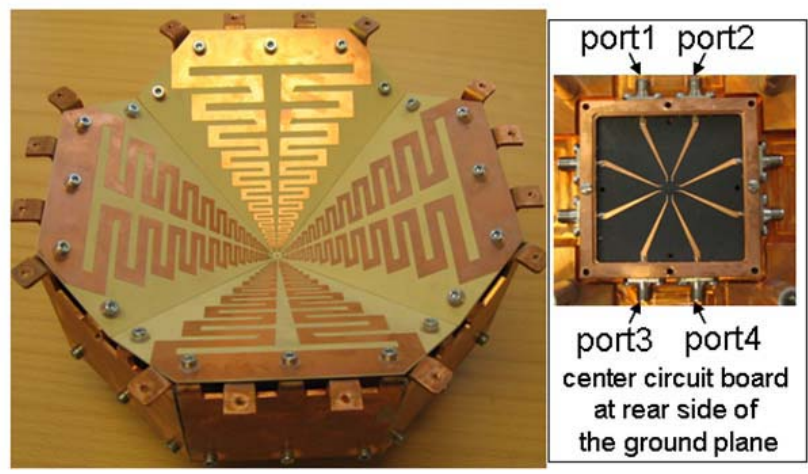

Fig. 2. Photos of front (left) and back (right) sides of Eleven antenna.

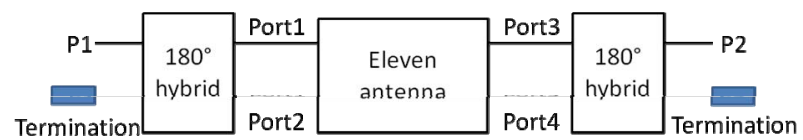

(a)

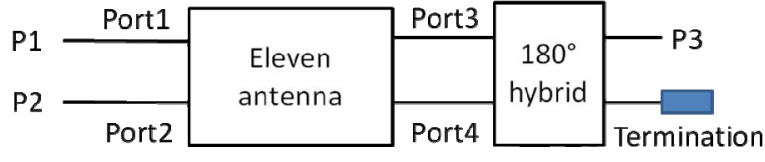

(b)

Fig. 3. Diagram of Eleven antenna with the four ports of one polarization combined to two-port antenna (a) and three-port antenna (b).

\section{MEASUREMENT AND RESULT}

\section{A. Measurement Setup}

In the measurement, the three wall antennas are used as transmit antennas, the AUT (i.e. Eleven antenna) is regarded as the multi-port receive antenna. During the measurement, the mode-stirrers moved step-wisely. At each stirrer position, the channel transfer function is sampled by a vector network analyzer (VNA). Due to the fact that the used VNA has a maximum frequency limit of $8.5 \mathrm{GHz}$, we decided to measure the Eleven antenna from 2 to $8 \mathrm{GHz}$. Since the channel coherence bandwidth inside the Bluetest chamber for this measurement setup is $1 \sim 2 \mathrm{MHz}$ [18], a 1-MHz frequency step was chosen. The VNA can gather a maximum of 1601 samples per frequency sweep. Therefore, we divided the whole measurement frequency range into four sub-bands, each with a sub-bandwidth of $1.5 \mathrm{GHz}$, i.e. $2-3.5 \mathrm{GHz}, 3.5-5 \mathrm{GHz}, 5-$ $6.5 \mathrm{GHz}$, and $6.5-8 \mathrm{GHz}$, and repeated the same measurement procedure over these four sub-bands. During the measurement procedure, the platform was moved to 20 positions spaced by $18^{\circ}$, and for each platform position each of the two plates moved simultaneously to 10 positions. At each stirrer position and for each of the three wall antennas a full frequency sweep was performed by the VNA. As a result, there are 200 MIMO channel samples per frequency point. In order to improve measurement accuracy, a $20-\mathrm{MHz}$ frequency stirring [19] is used (the frequency stirring is equivalent to treating uncorrelated or less correlated channel samples at different nearby frequencies as if they were spatial realizations of the same channel random process at the corresponding center frequency. The result is an increase of the equivalent independent sample number). Therefore, there are 4000 effective MIMO channel samples per frequency point after the frequency stirring.

For MIMO antenna characterizations, usually only shortterm fading is of interest. Therefore, normalization should be performed to calibrate out the frequency dependent path loss inside the chamber and at the same time preserve the antennas' imperfect efficiency and correlation effects. In order to do that, we repeat the measurement procedure again using a single-port reference disk-cone antenna [16] with known radiation efficiency. The reference level, $P_{\text {ref, }}$, was obtained by dividing the average power function by the radiation efficiency of the reference antenna (to calibrate out the reference antenna's imperfect radiation efficiency). Denoting the measured MIMO channel matrix as $\mathbf{H}$, the normalized measured channel matrix is

$$
\mathbf{H}_{\text {meas }}=\mathbf{H} / \sqrt{P_{\text {ref }}}
$$

where the reference level, $P_{r e f}$ is described above. Note that the total radiation efficiency of the wall antenna is also calibrated out by (1). Moreover, since the wall antennas were orthogonal polarized with sufficient separation (about 7 wavelengths at the lowest frequency), the correlations between them are negligible. Therefore the wall antennas can be regarded as ideal transmit antennas after the normalization (1).

\section{B. MIMO Capacity}

Throughout this paper, we assume that receiver has perfect channel state information (CSI) and transmitter has no CSI, and that the channel has flat fading and can be regarded as ergodic. The ergodic MIMO capacity can be estimated from the measured channel matrices by [1]

$$
\hat{C}=\frac{1}{N} \sum_{n=1}^{N}\left\{\log _{2}\left[\operatorname{det}\left(\mathbf{I}+\frac{\gamma}{N_{t}} \mathbf{H}_{\text {meas }}(n) \mathbf{H}_{\text {meas }}^{H}(n)\right)\right]\right\}
$$

where $\gamma$ is signal-to-noise ratio (SNR), the superscript ${ }^{H}$ is Hermitian operator, $\operatorname{det}(\cdot)$ denotes determinate, $\log _{2}$ denotes logarithmic function to the base 2, I is identity matrix, $N_{t}(=3)$ is the number of transmit (wall) antennas, $N(=4000)$ is the number of the samples per frequency point, and $n$ denotes the $n$th sample (or stirrer position). Note that flat fading assumption dictates that the ergodic capacity has to be calculated at each frequency point so that (2) holds.

To examine antenna effects on the ergodic capacity, we decompose $\mathbf{H}_{\text {meas }}$ using Kronecker channel model [1]

$$
\mathbf{H}_{\text {meas }}=\mathbf{R}_{r}^{1 / 2} \mathbf{H}_{w} \mathbf{R}_{t}^{1 / 2}
$$


where $\mathbf{R}_{t}$ and $\mathbf{R}_{r}$ are covariance matrices of the transmit and receive antennas respectively, $\mathbf{H}_{w}$ is independent and identically distributed (i.i.d.) channel matrix whose entries has zero mean and unity variance, and the superscript ${ }^{1 / 2}$ denotes Hermitian square root (or Cholesky decomposition) [20]. Note that the magnitude of the channel inside the reverberation chamber is Rayleigh distributed [21], thus

$$
\left.\operatorname{vec}\left(\mathbf{H}_{\text {meas }}\right) \sim C N\left(\mathbf{0}, \mathbf{R}_{t} \otimes \mathbf{R}_{r}\right)\right)
$$

where $\operatorname{vec}(\cdot)$ denotes the column-wise stacking of a matrix into a vector, $C N$ denotes complex Gaussian distribution, $\mathbf{0}$ is a vector of zeros, and $\otimes$ denotes Kronecker product. Although the Kronecker channel model is not accurate for large MIMOsize systems [4], it can be used to accurately model the channels of moderate MIMO-size systems [3], [6]. Moreover, it has been verified in [10] by comparing measured results in a reverberation chamber with Kronecker channel model results based on measurements in an anechoic chamber.

Since transmit (wall) antennas can be considered as ideal, $\mathbf{R}_{t}$ reduces to identity matrix. The receive antenna effects can be explicitly expressed via the covariance matrix

$$
\mathbf{R}_{r}=\boldsymbol{\Xi} \odot \boldsymbol{\Phi}
$$

where $\odot$ denotes entry-wise (Hadamard) product, and [22], [23]

$$
\begin{aligned}
& {[\mathbf{\Phi}]_{m n}=\frac{\iint_{4 \pi} \mathbf{g}_{m}(\Omega) \mathbf{P}_{i n c}(\Omega) \mathbf{g}_{n}^{*}(\Omega) d \Omega}{\sqrt{\iint_{4 \pi} \mathbf{g}_{m}(\Omega) \mathbf{P}_{i n c}(\Omega) \mathbf{g}_{m}^{*}(\Omega) d \Omega \cdot \iint_{4 \pi} \mathbf{g}_{n}(\Omega) \mathbf{P}_{i n c}(\Omega) \mathbf{g}_{n}^{*}(\Omega) d \Omega}},} \\
& \mathbf{\Xi}=\sqrt{\mathbf{e}} \sqrt{\mathbf{e}}^{T}, \\
& \mathbf{e}=\left[\begin{array}{llll}
e_{e m b 1} & e_{e m b 2} & \cdots & e_{e m b N_{r}}
\end{array}\right]^{T}
\end{aligned}
$$

where $[\cdot]_{m n}$ denotes the matrix entry with row index $m$ and

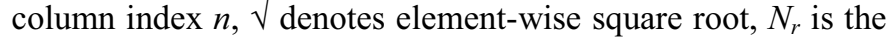
number of receive antennas, $\mathbf{g}_{n}$ is the embedded far field function (a vector with elements representing different polarizations) of the $n$th antenna port, $\Omega$ is the solid angle of arrival of the incident waves, and $\mathbf{P}_{i n c}$ is dyadic power angular spectrum of the incident waves. Note that in polarizationbalanced isotropic scattering environment, e.g. reverberation chambers, $\mathbf{P}_{i n c}(\Omega)=\mathbf{I}$.

Substituting (3) into (2) (note that $\mathbf{R}_{t}=\mathbf{I}$ ), we, after some mathematical manipulation, arrive at

$$
\hat{C}=\frac{1}{N} \sum_{n=1}^{N}\left\{\log _{2}\left[\operatorname{det}\left(\mathbf{I}+\frac{\gamma}{N_{t}} \mathbf{R}_{r} \mathbf{H}_{w}(n) \mathbf{H}_{w}^{H}(n)\right)\right]\right\} .
$$

It can be seen from (7) that, in a multipath environment like the reverberation chamber, the ergodic capacity depends on receive antenna only via its covariance matrix (5), which in turn depends on its embedded radiation efficiencies and correlation coefficients. Therefore, the embedded radiation efficiencies and correlation coefficients of the two- and threeport Eleven antennas are calculated and plotted in the next subsection along with the ergodic capacity.

\section{Measurement Results}

Due to the inherent symmetry property of the Eleven antenna (see Fig. 2), both ports of two-port Eleven antenna (see Fig. 3a) has approximately the same embedded radiation efficiency; the ports P1 and P2 of the three-port Eleven antenna (see Fig. 3b) has approximately the same embedded radiation efficiency, and the correlation coefficient (per frequency point) between P1 and P3 (of the three-port Eleven antenna) is approximately the same as that between P2 and P3.

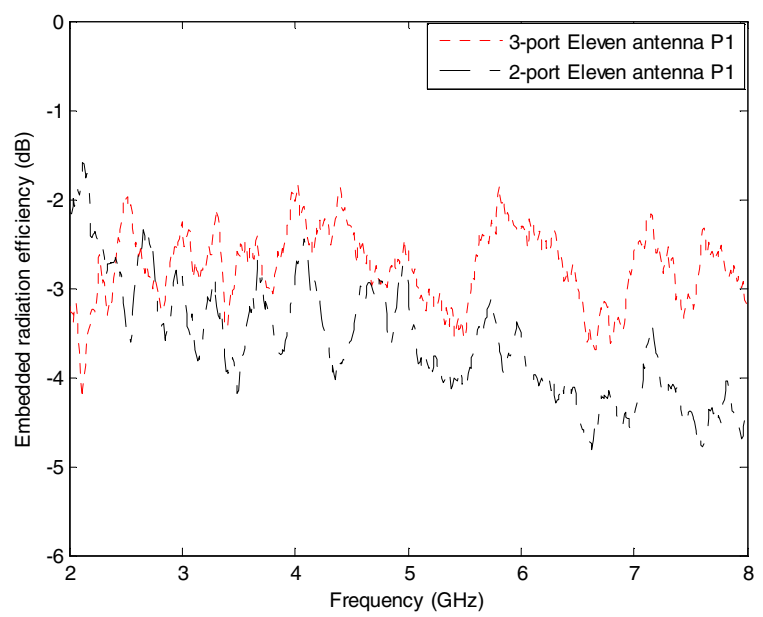

Fig. 4. Comparison of embedded radiation efficiencies of the two- and threeport Eleven antennas from measurements in reverberation chamber.

\section{1) Embedded radiation efficiency}

The embedded radiation efficiency of a port of the Eleven antennas can be measured in the reverberation chamber by the following procedure: first, the average power transfer function of a reference antenna with known radiation efficiency, $e_{r e f}$, is measured as $G_{r e f}$; then, the Eleven antenna is measured at the port of interest with another average power transfer function $G_{A U T}$. The corresponding embedded radiation efficiency is

$$
e_{e m b}=\frac{G_{A U T}}{G_{r e f} / e_{r e f}} .
$$

Note that the normalization factor, $P_{r e f}$, in (1) equals $G_{r e f} e_{r e f}$. Also note that the embedded radiation efficiency at P3 of the three-port Eleven antenna approximately equals to that at P1 of the two-port Eleven antenna (see Fig. 3). Hence, only the embedded radiation efficiencies at P1 ports of the two- and three-port Eleven antennas are plotted in Fig. 4. Note that the used $180^{\circ}$ hybrids (see Fig. 3 ) are the dominate contributor to the ohmic losses, and that is the reason that the embedded radiation efficiency at $\mathrm{P} 1$ of the three-port Eleven antenna (even though unmatched) is higher than that at P1 of the twoport Eleven antenna over most of the frequency range.

\section{2) Correlation coefficient}

The covariance matrix (in Rayleigh fading environments) can be estimated from the measured MIMO channels,

$$
\hat{\mathbf{R}}_{r}=\frac{1}{N N_{t}} \sum_{n=1}^{N} \mathbf{H}_{\text {meas }}(n) \mathbf{H}_{\text {meas }}^{H}(n) \text {. }
$$

The correlation coefficient between the $m$ th and $n$th ports can be estimated as 


$$
\hat{\rho}_{m n}=\frac{[\hat{\mathbf{R}}]_{m n}}{\sqrt{[\hat{\mathbf{R}}]_{m m}[\hat{\mathbf{R}}]_{n n}}} .
$$

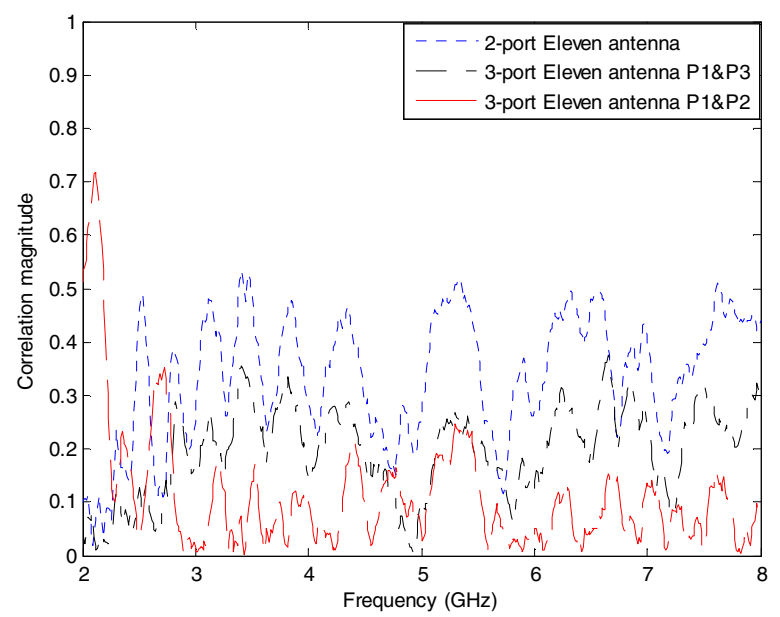

Fig. 5. Comparison of correlation coefficients of the two- and three-port Eleven antennas from measurements in reverberation chamber.

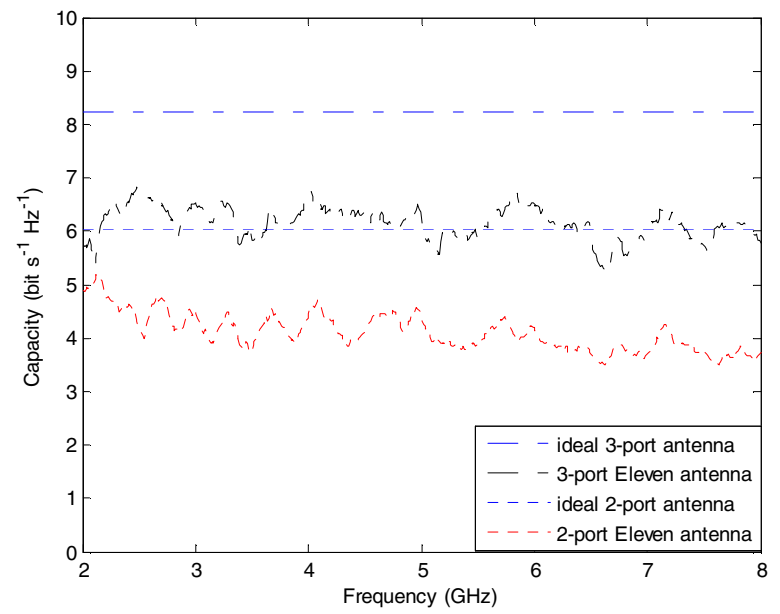

Fig. 6. Comparison of ergodic capacities of the two-port and three-port Eleven antennas from measurements in reverberation chamber at 10-dB SNR.

Note that the off-diagonal entries of $\hat{\mathbf{R}}_{r}$ are usually complex-valued, and that the diagonal entries are positive realvalued embedded radiation efficiencies of the corresponding ports. The correlation coefficients as a function of frequency are plotted in Fig. 5. It might be surprising to see that the correlation between the ports $\mathrm{P} 1$ and $\mathrm{P} 2$ of the three-port Eleven antenna (see Fig. 2 and Fig. 3) is lower than the other ones over most of the frequency range. This counter-intuition phenomenon is due to the fact that mutual couplings between antenna ports tend to make the embedded radiation pattern orthogonal, and therefore reduce correlations (see (6)) [24].

3) Ergodic capacity

The measured capacities at 10-dB SNR for the two-port and three-port Eleven antennas are shown in Fig. 6. For comparisons, the capacities of ideal (unity efficiency and no correlation) two-port and three port antennas are plotted in Fig. 6 as well. As expected, the three-port Eleven antenna has higher capacity than that of the two-port Eleven antenna, and that the corresponding ideal cases have higher capacities. Note that the capacity degradation for the two-port Eleven antenna is mainly due to the ohmic losses introduced by the $180^{\circ}$ hybrids, and for the three-port Eleven antenna it is due to the ohmic loss introduced by the $180^{\circ}$ hybrid and the mismatches at the ports $\mathrm{P} 1$ and $\mathrm{P} 2$.

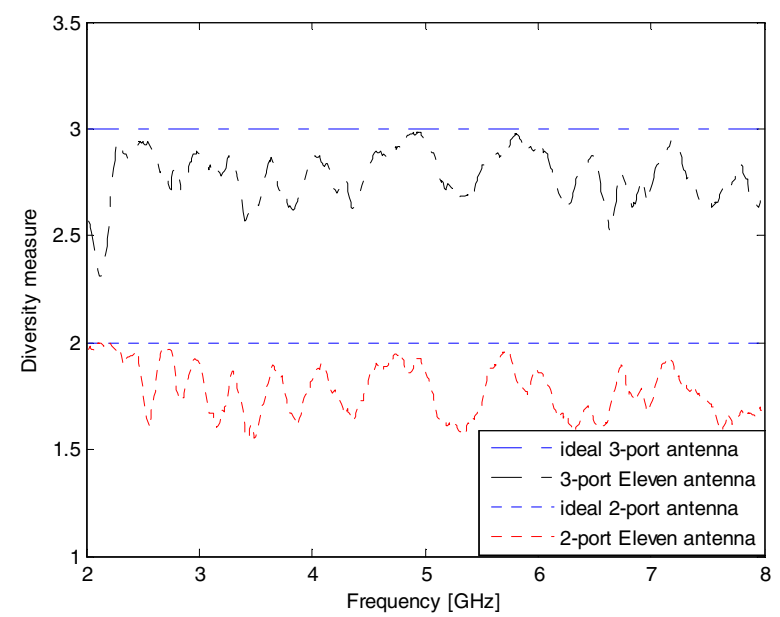

Fig. 7. Comparison of diversity measures of the two-port and three-port Eleven antennas from measurements in reverberation chamber.

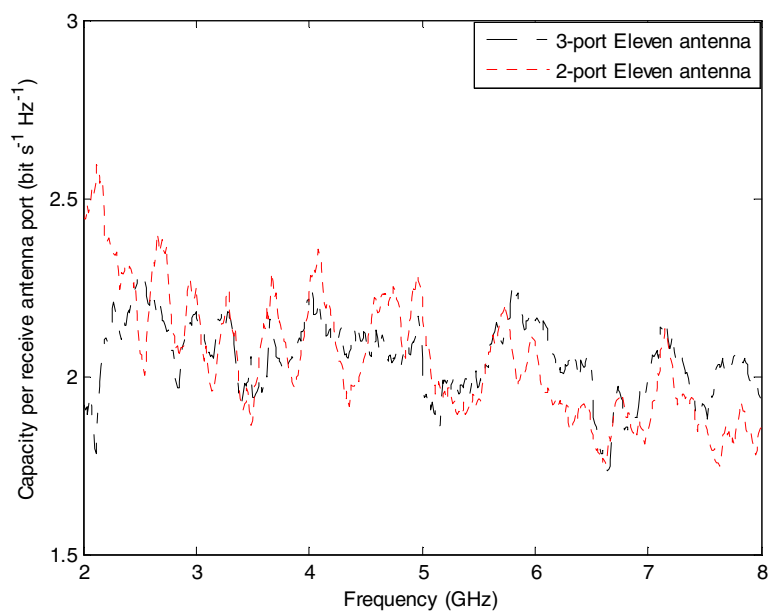

Fig. 8. Comparison of ergodic capacities per antenna port of the two-port and three-port Eleven antennas from measurements in reverberation chamber.

The ergodic capacity for MIMO antenna characterizations involves averaging over many channel realizations, and therefore suffers from computational overhead. An easy-tocompute parameter for MIMO antenna characterizations is the diversity measure defined as [25]

$$
\Psi\left(\mathbf{R}_{r}\right)=\left(\frac{\operatorname{tr}\left(\mathbf{R}_{r}\right)}{\left\|\mathbf{R}_{r}\right\|_{F}}\right)^{2}
$$

where $\operatorname{tr}(\cdot)$ is trace operator, and the subscript ${ }_{\mathrm{F}}$ denotes Frobenius norm. Like in the ergodic capacity formula (7), $\mathbf{R}_{r}$ is the sufficient statistic [2] of the antenna for the diversity measure. Fig. 7 shows diversity measures of the two- and three-port Eleven antenna together with their corresponding ideal cases. As expected, given fixed transmit antennas, a 
larger diversity measure of the multi-port receive antenna implies a larger ergodic MIMO capacity.

The superior capacity performance of the three-port Eleven antenna to the two-port Eleven antenna is at the cost of an additional RF chain, and it might not be a fair comparison for MIMO performance characterization. Thus we also plotted the capacity per receive antenna port, $\hat{C} / N_{r}$, in Fig. 8 . It is seen that the capacity per antenna port of two-port and threeport Eleven antennas are approximately the same. Therefore, the superior capacity performance of the three-port Eleven antenna is mainly due to the additional RF chain.

\section{CONCLUSION}

In this paper, we showed that the Eleven antenna can be configured into different multi-port antennas using different feeding networks. The MIMO performance of two multi-port Eleven antenna configurations were studied by measurements in a reverberation chamber. Their embedded radiation efficiencies, correlation, diversity measure, and ergodic capacity were compared in details. The Eleven antenna is shown to be a strong reference MIMO antenna candidate for various over-the-air MIMO tests.

\section{REFERENCES}

[1] Paulraj, R. Nabar and D. Gore, Introduction to Space-time Wireless Communication, Cambridge university press, 2003.

[2] T. M. Cover and J. A. Thomas, Elements of Information Theory, John Wiley \& Sons, 1991.

[3] K. Yu, M. Bengtsson, B. Ottersten, D. McNamara, P. Karlsson and M. Beach, "Modeling of wide-band MIMO radio channels based on NLoS indoor measurements," IEEE Trans. Vehic. Technol. vol. 53, no. 3, pp. 655-665, May 2004

[4] H. Ozcelik, M. Herdin, W. Weichselberger, J. Wallace and E. Bonek, "Deficiencies of 'Kronecker' MIMO radio channel model," Electron. lett., vol. 39, no. 16, pp. 1209-1210, Aug. 2003.

[5] J. W. Wallace, M. A. Jensen, A. L. Swindlehurst, and B. D. Jeffs, "Experimental characterization of the MIMO wireless channel: data acquisition and analysis," IEEE Trans. Wireless Commun., vol. 2, no. 2, pp. 335-342, Mar. 2003

[6] J. P. Kermoal, L. Schumacher, K. I. Pedersen, P. E. Mogensen, and F. Frederiksen, "A stochastic MIMO radio channel model with experimental validation," IEEE J. Sel. Areas Commun., vol. 20, pp. 1211-1226, Aug. 2002.

[7] D. Chizhik, J. Ling, P. W. Wolniansky, R. A. Valenzuela, N. Costa and K. Huber, "Multiple-input-multiple-output measurements and modeling in Manhattan," IEEE J. Sel. Areas Commun., vol. 21, no. 3, pp. 321-330, Apr. 2003.

[8] K. Rosengren and P-S. Kildal, "Radiation efficiency, correlation, diversity gain and capacity of a six monopole antenna array for a MIMO System: theory, simulation and measurement in reverberation chamber," in Proc. IEE, Microwaves, Optics Antennas, pp. 7-16, Vol.152, No.1, Feb 2005. (See also Erratum, Aug. 2006.)
[9] P.-S. Kildal and K. Rosengren, "Correlation and Capacity of MIMO Systems and Mutual Coupling, Radiation Efficiency, and Diversity Gain of Their Antennas: Simulations and Measurements in a Reverberation Chamber", IEEE Communications Magazine, vol. 42, no. 12, pp. 102112, Dec. 2004.

[10] X. Chen, P.-S. Kildal, J. Carlsson, and J. Yang, "Comparison of ergodic capacities from wideband MIMO antenna measurements in reverberation chamber and anechoic chamber," IEEE Antennas Wireless Propag. Lett., vol. 10, pp. 446-449, 2011.

[11] X. Chen, P.-S. Kildal, J. Carlsson, "Fast converging measurement of MRC diversity gain in reverberation chamber using covarianceeigenvalue approach," IEICE Transactions on Electronics, vol.E94C, no.10, pp.1657-1660, Oct. 2011.

[12] J. F. Valdes, M. A. Fernandez, A. M. Gonzalez, and D. A. Hernandez, "The influence of efficiency on receive diversity and MIMO capacity for Rayleigh-fading channels," IEEE Trans. Antennas Propag., vol. 56, no. 5, pp. 1444-1450, May 2008.

[13] O. Delangre, P. D. Doncker, M. Lienard and P. Degauque, "Coupled reverberation chamber for emulating MIMO channels," C. R. Physique 11, pp. 30-36, 2010

[14] L. Garcia-Garcia, B. Lindmark, N. Jalden and C. Orlenius, "MIMO capacity of antenna arrays evaluated using radio channel measurements, reverberation chamber and radiation patterns," IET Microw. Antennas Propag., vol. 1, pp. 1160-1169, 2007.

[15] P.-S. Kildal, A. Hussain, X. Chen, et al., "Threshold receiver model for throughput of wireless devices with MIMO and frequency diversity measured in reverberation chamber," IEEE Antennas Wireless Propag. Lett., vol. 10, pp. 1201-1204, 2011.

[16] C. A. Balanis, Antenna theory: Analysis and Design. 3rd ed., John Wiley \& Sons, 2005

[17] J. Yang, M. Pantaleev, P.-S. Kildal, Y. Karadikar, L. Helldner, B. Klein, N. Wadefalk, C. Beaudoin, "Cryogenic 2-13 GHz eleven feed for reflector antennas in future wideband radio telescopes", IEEE Trans. Antennas Propag., vol. 59, no. 6, pp. 1918-1934, June, 2011.

[18] X. Chen, P.-S. Kildal, C. Orlenius, and J. Carlsson, "Channel sounding of loaded reverberation chamber for Over-the-Air testing of wireless devices - coherence bandwidth and delay spread versus average mode bandwidth," IEEE Antennas Wireless Propag. Lett., vol. 8, pp. 678-681, 2009.

[19] D. A. Hill, "Electronic mode stirring for reverberation chamber", IEEE Trans. Electromagn. Compat., vol. 36, no.4, pp. 294-299, Nov. 1994.

[20] A. J. Laub, Matrix analysis for scientists and engineers: Siam, 2005.

[21] J. G. Kostas and B. Boverie, "Statistical model for a mode-stirred chamber," IEEE Trans. Electromagn. Compat., vol. 33, no. 4, pp. 366370, Nov. 1991.

[22] R. G. Vaughan and J. B. Andersen, "Antenna diversity in mobile communications", IEEE Trans. Vehic. Technol. vol. 36, no. 4, pp. 149172, Nov., 1987.

[23] X. Chen, P.-S. Kildal, J. Carlsson, "Measurement uncertainties of capacities of multi-antenna system in anechoic chamber and reverberation chamber," in Proc. $8^{\text {th }}$ Int. Symp. Wireless Commun. Systems (ISWCS'11), Aachen, Germany, Nov. 2011.

[24] X. Chen, P.-S. Kildal, J. Carlsson, "Comparisons of different methods to determine correlation applied to multi-port UWB eleven antenna," in Proc. 5th Eur. Conf. Antenna Propag., Rome, Italy, Apr. 2011, pp. 1776-1780.

[25] M. T. Ivrlac and J. A. Nossek, "Diversity and correlation in Rayleigh fading MIMO channels," in Proc. IEEE VTC, May 2005, pp. 151-155. 